\title{
The pro-apoptotic role of autophagy in breast cancer
}

\author{
S Suman ${ }^{1,3,4}$, T P Das $^{1,3,4}$, R Reddy ${ }^{1}$, A M Nyakeriga ${ }^{1}$, J E Luevano ${ }^{1}$, D Konwar ${ }^{2}$, P Pahari ${ }^{2}$ and \\ C Damodaran ${ }^{\star}, 1,3$ \\ ${ }^{1}$ Department of Biomedical Sciences, Paul L Foster school of Medicine, Texas Tech University Health Sciences center, El Paso, \\ TX, USA; ${ }^{2}$ Division of Synthetic Organic Chemistry, CSIR-North East Institute of Science and Technology, Jorhat 785006, Assam, \\ India and ${ }^{3}$ Department of Urology, University of Louisville, KY 40202, USA
}

Background: Autophagy is a catabolic process that has a vital role in cancer progression and treatment. Current chemotherapeutic agents, which target autophagy, result in growth inhibition in many cancer types. In this study, we examined the role of autophagy in breast cancer (BCa) patients as well as BCa cell lines.

Methods: Tissue microarray was used to detect the expression of an autophagy marker, LC3B in BCa patients (normal/ hyperplasia $=8$; grade- $\mathrm{I}=15$, grade- $I I=84$, and grade- $I I=27$ ) and $B C$ a cell lines. To modulate the activation of autophagy, we used novel herbal compound nimocinol acetate (NA) in BCa cell lines and the anticancer activity was measured by phenotypic and molecular analysis.

Results: LC3B is highly expressed in tumours as compared with normal tissues. Activation of LC3B in NA-treated BCa (MCF-7 and MDA-MB-231) cells was evident as compared with other autophagy makers. Further, our results confirmed that NAtranscriptionally regulates LC3B (as confirmed by mRNA levels and reporter assay), which resulted in the formation of acidic autophagy vesicles and autolysosomes in BCa cells. Nimocinol acetate inhibited mTOR-mediated pro-survival signalling that resulted in inhibition of growth in BCa cells without affecting normal breast epithelial cells. Downregulation of LC3B expression by siRNA significantly inhibited the anticancer effects of NA in BCa cells.

Conclusions: Together, our results suggest that LC3B is highly expressed in BCa tissues and increasing the threshold of LC3B activation dictates the pro-apoptotic function, which in turn, suppresses the growth of BCa cells. Nimocinol acetate could be a potential agent for treatment of $\mathrm{BCa}$.

Autophagy, a lysosomal degradation process, removes and recycles damaged organelles to maintain the quality of intracellular components. The role of autophagy is complex. Studies have shown that autophagy promotes cancer cell survival by protecting cells from hypoxia, starvation, oxidative stress, and by causing chemoresistance in established tumours (Kroemer and Jaattela, 2005; Kondo and Kondo, 2006; Kroemer and Levine, 2008). Autophagy-mediated chemoresistance was reported for anticancer agents such as camptothecin in breast cancer $(\mathrm{BCa})$ cells and for cisplatin in colon and lung cancer cells (Abedin et al, 2007; Li et al, 2010; Ren et al, 2010). In contrast, the anticancer agents temozolomide and dexamethasone, radiation, and pharmacological inhibitors have been shown to induce autophagy as the primary action that promotes growth arrest in cancer cells (Paglin et al, 2001; Kanzawa et al, 2004; Zeng and Kinsella, 2008; Laane et al, 2009). Hence, these studies clearly suggest the pivotal role of autophagy in cancer, but still the molecular switch or mechanism that dictates autophagy-mediated survival or death of the cell is unknown. Furthermore, the diverse functions of autophagy may depend on the specific cell types, tumour microenvironment, and the duration of treatment.

Autophagy is regulated by several molecular events. Although it has been well established that the activation of mammalian target of rapamycin (mTOR) negatively regulates autophagy, in a number

*Correspondence: Dr C Damodaran; E-mail: Chendil.Damodaran@louisville.edu

${ }^{4}$ These authors contributed equally to this work.

Received 23 December 2013; revised 13 March 2014; accepted 18 March 2014; published online 19 June 2014

(c) 2014 Cancer Research UK. All rights reserved 0007-0920/14 
of preclinical studies, rapamycin, an mTOR inhibitor, has been shown to induce growth arrest by activating autophagy (Ravikumar et al, 2004). Activated mTOR signalling leads to phosphorylation of the serine/threonine-protein kinase of the autophagy-related gene (ATG)/ULK1 complex, a key regulator of autophagy. ATG/ULK1 is required to initiate the autophagasome formation that eventually promotes autolysosomal degradation through the activation of light chain 3A (LC3A) (Hara et al, 2008; Hosokawa et al, 2009; Jung et al, 2009). In mammalian cells, three types of LC3 isoforms were reported; A, B, and C, however, LC3B has an important role in the formation of autophagosome, and it is considered to be a valid marker for autophagy in cancer cells (Klionsky and Emr, 2000).

Some of the ATG genes act as tumour suppressors at the earlier stages of tumourigenesis. Once a tumour is established, oxidative stress and tumour microenvironments may activate the functions of the autophagy gene products by initiating the pro-survival machinery in cancer cells (recently reviewed in (Yang et al, 2012). In support of the tumour suppressive role of ATG genes, several studies suggested that the beclin 1 gene was deleted in ovarian (Russell et al, 1990), breast (Futreal et al, 1992; Liang et al, 1999), and brain tumours (Miracco et al, 2007). Further, it was demonstrated that $\mathrm{BCa}$ cell lines contained deletions of one allele in beclin 1 (ATG6), indicative of compromised function of autophagy in cancer (Liang et al, 1999). Similarly, in mouse models of cancer, the heterozygous disruption of the mouse beclin 1 gene promoted tumourigenesis (Qu et al, 2003).

Autophagy is considered to be a second type of programmed cell death (Lum et al, 2005). The molecular signalling for both autophagy and apoptosis are interconnected, as both share and target mitochondrial degradation that results in inhibition of several pro-survival signalling molecules, including $\mathrm{Bcl}-2$ and $\mathrm{BCL}_{\mathrm{xL}}$. These pro-survival genes bind to beclin 1, and form a trimeric complex, which inhibits beclin 1-mediated autophagy function, resulting in proliferation and survival of cancer cells (Furuya et al, 2005; Pattingre et al, 2005).

$\mathrm{BCa}$ remains the second leading cause of cancer deaths in the United States and worldwide (Siegel et al, 2013). Multiple therapeutic agents, along with radiation and surgery, are the current standard therapeutic regimen for $\mathrm{BCa}$ patients. Although BCa mortality has steadily decreased owing to early detection and availability of effective therapeutic options, novel therapeutic targets and potent molecules are still needed to completely eradicate BCa. It is known that naturally occurring molecules exhibit cancer-preventive and therapeutic properties by inducing cell cycle arrest, inhibiting the pro-survival machinery, activating tumour suppressor genes, and causing induction of apoptosis, necrosis, or autophagy in BCa models. We have identified a novel anticancer agent, nimocinol acetate (NA) (Supplementary Figure 1), from the edible fruit of Chisocheton paniculatus, which is grown in tropical regions. Previous studies suggest that paniculatus extract exhibits antiinflammatory (Miller et al, 2008), antimicrobial, antimalarial, antifungal, and cytotoxic activities (Chen et al, 2012; Sharma et al, 2012; Shi et al, 2012).

In this study, we demonstrate LC3B is highly expressed in breast tumour samples as compared with normal breast tissues. Autophagy is the primary process affected by a natural compound, NA, and NA treatment causes growth inhibition in both oestrogen-responsive and oestrogen-refractory BCa cell lines. Molecular analysis suggested that LC3B may be a target of NA. Nimocinol acetate specifically affected $\mathrm{BCa}$ cells without causing toxicity to normal breast epithelial cells, a property that may aid the application of NA in clinical settings.

\section{MATERIALS AND METHODS}

Cell lines and reagents. BCa cell lines (MCF-7 and MDA-MB231) were purchased from the American Type Culture Collection (Manassas, VA, USA). MCF-7 and MDA-MB-231 cells were maintained in Dulbecco's modified Eagle's medium (DMEM) containing L-glutamine, sodium pyruvate, supplemented with $10 \%$ fetal bovine serum and $1 \%$ antibiotic and anti-mycotic solution, in a humidified atmosphere of $5 \% \mathrm{CO}_{2}$ at $37^{\circ} \mathrm{C}$. Human mammary immortalised cells (MCF-12A) were maintained in $1: 1$ mixture of DMEM and Ham's F12 medium with $20 \mathrm{ng} \mathrm{ml}^{-1}$ human epidermal growth factor, $100 \mathrm{ng} \mathrm{ml}^{-1}$ cholera toxin, $0.01 \mathrm{mg} \mathrm{ml}^{-1}$ bovine insulin, $500 \mathrm{ng} \mathrm{ml}^{-1}$ hydrocortisone, and $5 \%$ horse serum. Blood samples were obtained from healthy volunteers through the United Blood Services (El Paso, TX, USA) and the procedures were reviewed and approved by the Texas Tech University IRB committee. Human normal peripheral blood mononuclear cells (PBMCs) were isolated by Ficoll-Paque centrifugation. Peripheral blood mononuclear cells were seeded in six-well plates in serumfree RPMI-1640. Nimocinol acetate was identified and synthesised in the laboratory of Dr Pallab.

Immunohistochemical analysis of human BCa tissue array. For immunohistochemical analysis human BCa TMA (Cat no. BRC1509) was purchased from Pantomics (Richmond, CA, USA). For each grade the TNM classification is provided in the product data sheet. BCa tissue array slide was stained with primary antibody for LC3B, followed by secondary antibody incubation, and was analysed under a light microscope. The tissue microarray (TMA) slide was viewed and scored by a pathologist.

Cell viability assay. Cells were treated with various concentrations of NA, or dimethyl sulphoxide (DMSO; vehicle, referred as untreated-UT in all the figures) for $24 \mathrm{~h}$. Cell viability was quantified using the trypan blue exclusion method and cell proliferation was assessed by Bromodeoxyuridine (BrdU) incorporation assay (Cell Signaling, Danvers, MA, USA), according to the manufacturer's protocol (Suman et al, 2013b).

Apoptosis assay. Apoptosis assays were performed with the Annexin V-fluorescein isothiocyanate apoptosis kit-I (BD Pharmingen, San Diego, CA, USA) as described previously (Das et al, 2013).

Soft agar colony-formation assay. A colony-formation assay was performed to monitor anchorage-independent growth using the CytoSelect 96-Well In Vitro Tumor Sensitivity Assay kit (Cell Biolabs Inc., San Diego, CA, USA). MCF-7, MDA-MB-231, and MCF-12 A cells $\left(5 \times 10^{3}\right)$ were harvested and assayed as described earlier (Suman et al, 2013b).

Protein extraction and western blotting. BCa cell lines were seeded in six-well plates, and after $24 \mathrm{~h}$ of culture, the cells were treated with NA at different dose and time. Western blotting was performed using specific antibodies against, Atg3, Atg5, Atg7, Atg12, beclin 1, LC3A, LC3B (Autophagy antibody sampler kit \#4445, Cell Signaling), mTOR (\#2972), pmTOR (\#2974), p70S6K (\#2708), phospho-p70S6K (\#9234), BAX (\#2772), BCL-2 (\#2876), cleaved caspase-3 (\#9661), cleaved caspase-9 (\#9505), PARP (\#9542) and cleaved PARP (\#9541) (Cell Signaling), and $\beta$-actin (Santa Cruz Biotechnology, Dallas, TX, USA). Protein-antibody complexes were detected with the enhanced chemiluminescence method as described earlier (Suman et al, 2013b).

Construction of Human LC3B luciferase reporter gene constructs. MAP1LC3B (microtubule-associated protein 1 light chain 3 beta) is a protein-coding gene with gene ID: 81631 . The reporter construct LC3B-luc contains -1000 to +1 base pairs of the human $L C 3 B$ gene inserted upstream of a firefly luciferase 
reporter gene in pGL3 basic vector (Promega, Madison, WI, USA). For construction of the 1000 -bp $(-1000$ to +1$)$ reporter construct, a forward primer $5^{\prime}$-CAGGGTACCTATGACTGCCCTTTCACAGA-3', containing $K p n \mathrm{I}$ site at the $5^{\prime}$ end, and a reverse primer 5'-CAGCTCGAGCATGGTGCAGGGATCTGGGC- $3^{\prime}$, containing XhoI site at the $5^{\prime}$ end were used for PCR. Normal genomic DNA was taken as a template. The PCR product was digested with $K p n I$ and XhoI enzymes and cloned into PGL3-basic luciferase vector and sequenced to confirm the orientation and insert (Supplementary Figure 2).

Dual-luciferase reporter assay. To perform the dual-luciferase reporter assay, LC3B-luc promoter constructs $(2 \mu \mathrm{g})$ and Renilla luciferase $(100 \eta \mathrm{g})$ vectors were cotransfected using Lipofectamine 2000 (Invitrogen, Grand Island, NY, USA). The reporter assay was performed using the Dual-Luciferase Reporter Assay System from Promega.

siRNA transfection. BCa cell lines were seeded in six-well plates at a density of $2 \times 10^{5}$ cells per well. After $24 \mathrm{~h}$ of culture, the cells were transiently transfected with short interfering RNA (siRNA) specific for LC3B (siLC3B), or a control siRNA, as described earlier (Suman et al, 2013a).

Immunofluorescence analysis. BCa cell lines were seeded on glass coverslips and allowed to attach and grow to $60 \%$ confluence as described earlier (Das et al, 2013). Following treatment with NA for $24 \mathrm{~h}$, cells were washed and then incubated with LC3B antibody followed by secondary antibodies conjugated to Alexa Fluor 488 (green) to detect the localisation and expression of LC3B. Cells were analysed using a Nikon laser scanning confocal microscope (Nikon Instruments Inc., Melville, NY, USA).

Analysis of autophagosome and autolysosome formation. To analyse NA-induced autophagosome and autolysosome formation over time in MCF-7, MDA-MB-231, and MCF-12A cells, we used the commercially available Premo Autophagy Tandem Sensor RFP-GFP-LC3B Kit (Life technologies, Carlsbad, CA, USA). All three cell lines were plated in $35-\mathrm{mm}$ dishes and allowed to reach $70 \%$ confluence by culturing for $24 \mathrm{~h}$ before NA treatment. All cells were simultaneously transduced with the RFP-GFP-LC3B reagent and treated with NA for $48 \mathrm{~h}$. The cells were visualised with a live cell-imaging microscope using standard green fluorescent protein (GFP) and red fluorescent protein (RFP) settings. The neutral $\mathrm{pH}$ autophagosome, identified by GFP emission, and acidic autolysosome, identified by RFP emission, were visualised every $1 \mathrm{~h}$ for up to $48 \mathrm{~h}$.

Statistical analysis. Statistical analysis was performed using Graphphad prism 6.0a software (GraphPad software Inc., La Jolla, CA, USA). The results are represented as the mean \pm s.e.m. Data sets were compared using a two-tailed unpaired Student's $t$-test. Statistical significance was set at $P<0.05$.

\section{RESULTS}

Human BCa tissue array showed high expression of LC3B in higher tumour grades. LC3B is considered as an important marker for autophagy as it is involved in autophagosome formation and has a vital role in BCa. To analyse the expression pattern of LC3B, immunohistochemical studies were performed on human breast cancer TMA. Breast cancer patients (normal (representing hyperplasia or fibroadenoma $)=8$; grade- $\mathrm{I}=15$, grade- $\mathrm{II}=84$, and grade-III $=27$ ) were studied. The scoring of TMA revealed high expression of LC3B in tumours as compared with normal tissues (Figures $1 \mathrm{~A}$ and $\mathrm{B}$ ), suggesting the important role of $\mathrm{LC} 3 \mathrm{~B}$ in human $\mathrm{BCa}$.
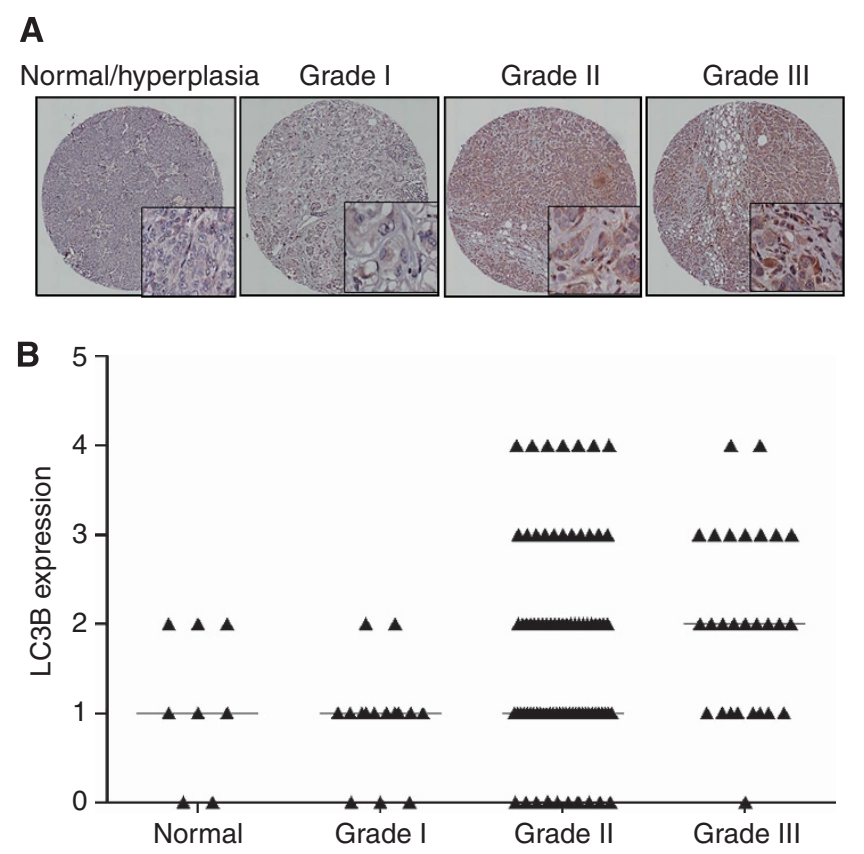

Figure 1. Human $\mathrm{BCa}$ tissue array demonstrating the expression of LC3B in BCa patients. (A) Immunoreactivity for LC3B in representative sections of human BCa. (B) Scatter plot representing LC3B expression score based on labelling intensity and given as median.

NA specifically induces growth inhibition in BCa, and not normal breast epithelial, cells. To evaluate the anticancer effect of NA on BCa cells, cell viability (trypan blue exclusion), proliferation (BrdU incorporation) and soft agar colony-formation assays were performed on ER-positive (MCF-7), ER-negative (MDA-MB-231), and normal breast epithelial (MCF-12A) cells. Nimocinol acetate inhibited the proliferation of both MCF-7 and MDA-MB-231 cells in a dose-dependent manner at $24 \mathrm{~h}$ (Figure 2A). The $\mathrm{IC}_{50}$ values for both MCF-7 and MDA-MB231 cells were 5 and $7.5 \mu \mathrm{M}$, supporting the inhibitory role of NA on $\mathrm{BCa}$ cells. Colony-formation assays further supported the inhibitory effect of NA on the growth of BCa cells (Figure 2B). Oestrogen-refractory $\mathrm{BCa}$ cells are more aggressive and highly proliferative, and promotion of growth inhibition by NA suggests that, regardless of the ER status, NA could be a potent agent against BCa.

Next, we determined the toxicity of NA on normal breast epithelial cells and PBMCs. Interestingly, no significant growth inhibition was observed in normal breast epithelial-MCF-12A cells treated with various concentrations of NA (Figure 2C). However, $20 \%$ inhibition of cell viability was seen at the higher concentration $(10 \mu \mathrm{M})$ of NA in PBMCs (Supplementary Figure 3 ). It is of note that as the $\mathrm{IC}_{50}$ values for both $\mathrm{BCa}$ cells were approximately $5-7.5 \mu \mathrm{M}$, NA appears to target BCa cells than normal breast epithelial cells. To investigate whether the NA-induced changes in BCa cells were due to induction of apoptosis or cell cycle arrest, we evaluated the induction of apoptosis and cell cycle regulation after treatment with NA by flowcytometry analysis. No significant induction of apoptosis was seen at the $\mathrm{IC}_{50}$ drug concentration in either of the NA-treated BCa cell lines (Supplementary Figure 4A). Similarly, no changes in the proportion of cells at the $G_{0} / G_{1}, G_{2} / M$, or $S$ phases of the cell cycle were observed in $\mathrm{BCa}$ cell lines treated either with the vehicle (DMSO) or NA (Supplementary Figures $4 \mathrm{~B}$ and $4 \mathrm{C}$ ). These results suggest that NA-induced growth inhibition was not due to induction of apoptosis or changes in cell cycle regulation. Therefore, we hypothesised that autophagy might be involved in the NA-induced reduction of cell viability in BCa cells. 

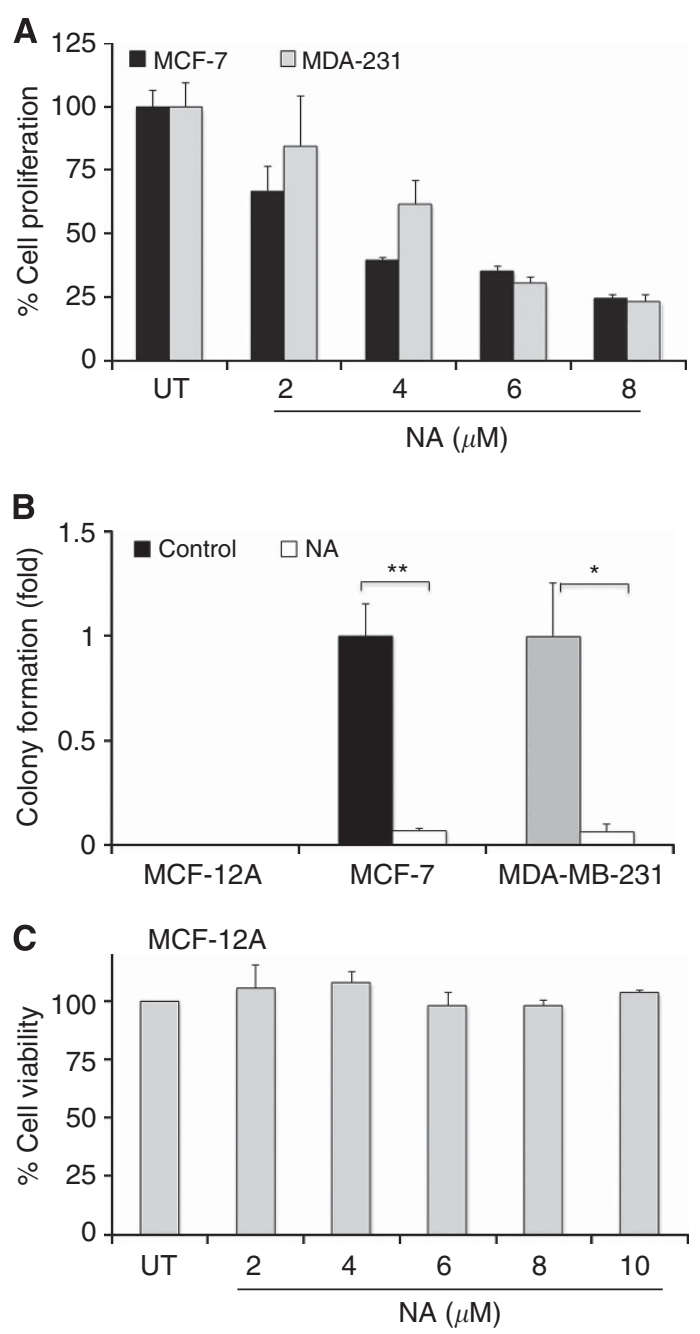

Figure 2. Induction of growth inhibition by NA in BCa cells, but not in normal cells. (A) MCF-7 and MDA-MB-231 cells were treated with indicated concentrations of NA or DMSO (vehicle) for $24 \mathrm{~h}$ and cell proliferation was assessed by BrdU incorporation-based cell proliferation assay. (B) Anchorage-independent growth was measured by the colony-formation assay. (C) Effect of NA on normal breast epithelial cells (MCF-12A) was measured by the trypan blue exclusion assay. Cells were treated with indicated concentrations of NA for $24 \mathrm{~h}$ followed by analysis of cell proliferation using the trypan blue exclusion assay. ${ }^{\star} P<0.05$ and ${ }^{*} P<<0.005$.

NA induces the expression of autophagy markers in BCa cells. While analysing the anticancer effects of NA, we observed morphological changes in $\mathrm{BCa}$ cell lines treated with $\mathrm{NA}$ as compared with untreated cells. In particular, many changes were evident when visualising the autophagic vacuoles by phase-contrast microscopy in NA-treated cells (data not shown). So, we investigated the kinetics of the induction of autophagy markers, including the Atg family of proteins, in NA-treated BCa cells. Atg3 expression was significantly induced in a time- and dosedependent manner in NA-treated BCa cells, as compared with two other ATG gene family proteins, that is, Atg7 and Atg12 (Figures 3A and C). Treatment with NA moderately induced Atg5 expression in both BCa cells. Beclin 1 is a known ATG family tumour suppressor involved in autophagy; so, we evaluated whether NA upregulates Beclin 1 expression. Our data showed that beclin 1 levels remained unaltered between control and NAtreated cells (Figures 3B and D).

We next evaluated an important autophagy marker, LC3B, a microtubule-associated protein, which has an critical role in autophagy, especially in the formation of the autophagosome (Mizushima and Yoshimori, 2007). During autophagy induction, LC3-I, a cytosolic form of LC3, is converted to LC3-II by lipidation, which facilitates its binding with the membranes of autophagosomes (Kabeya et al, 2000). Treatment with NA upregulated LC3A (A-I and A-II) and LC3B (B-I and B-II) expression in a dose- and timedependent manner in $\mathrm{BCa}$ cell lines (Figures $3 \mathrm{~B}$ and $\mathrm{D}$ ). However, a significant induction of LC3B was seen as compared with LC3A in both BCa cell lines. Further, NA activates LC3B promoter in MCF-7 (5.4-fold) and MDA-MB-231 (4.2-fold) cells (Figure 3E). In addition, we also confirmed a transcriptional regulation of LC3B in both cell lines (Supplementary Figure 5).

Induction of autophagosome and autolysosome in NA-treated BCa cells. As NA upregulates LC3B expression, and LC3B is a known marker for autophagosomes, we examined the localisation of LC3B in NA-treated BCa cells using confocal microscopy. BCa cell lines displayed a significant increase in LC3B-positive puncta upon treatment with NA (Figures 4A and B). MDA-MB-231 cells had more LC3B-positive puncta (61\%) and these puncta were larger, compared with those in MCF-7 cells (52\%) (Figures 4A and B). Interestingly, in MDA-MB-231 cells, NA-induced puncta were seen near perinuclear sites. These results implied the recruitment of LC3B in the formation of autophagosomes in NA-treated BCa cells.

Next, the formation of autophagosomes and autolysosomes was visualised using a Premo autophagy tandem sensor kit. Using a chimeric protein, with LC3B tethered to an acid-sensitive GFP and an acid-insensitive RFP, we were able to detect the formation of autophagosomes. Autophagosomes exhibit neutral $\mathrm{pH}$ (allowing emission of fluorescence by both RFP and GFP) and autolysosome formation produces acidic $\mathrm{pH}$ (which allows emission of fluorescence by RFP), allowing the visualisation of these subcellular structures using a live cell-imaging microscope. Brighter GFP signals were seen at $6 \mathrm{~h}$ followed by the RFP-specific emission, and more intense fluorescence emission was seen at $24 \mathrm{~h}$, and persisted up to $48 \mathrm{~h}$, in MCF-7 cells, as compared with MDA-MB-231 cells. Although we expected a similar intensity of GFP and RFP in MDA-MB-231 cells, due to aggressive growth of the MDA-MB-231 cells, the cells migrated from the origin as the time progressed (please see the Supplementary Figure 6 in Supplementary Material). In MCF-12A cells, the GFP and RFP signals were of much lower intensity, suggesting that NA-induced autophagosome and autolysosome formation occurred only in BCa cells, and not in normal breast epithelial cells (Figure 4C).

NA inhibits the phosphorylation of mTOR and simultaneously induces pro-apoptotic signalling in BCa cells. Activation of mTOR inhibits autophagy that resulted in proliferation of cancer cells (Kondo and Kondo, 2006). Inhibition of mTOR signalling by rapamycin activates autophagy that in turn causes growth arrest in many preclinical models of cancer (Ravikumar et al, 2004). On the basis of these effects of mTOR signalling on autophagy, we determined whether NA inhibits mTOR activation in MCF-7 and MDA-MB-231 cells. Nimocinol acetate downregulated the expression of phospho-mTOR in a time-dependent manner in both $\mathrm{BCa}$ cells (Figure 5A). Similarly, the levels of phospho-p70S6K, a serine/ threonine kinase that is involved in $\mathrm{mTOR}$ signalling, decreased upon treatment with NA in a time-dependent manner (Figure 5A). These results suggest that NA inhibits mTOR signalling in BCa cells.

As Bcl-2 and BCL $\mathrm{BL}_{\mathrm{X}}$ in complex with beclin1 have been shown to inhibit autophagy in many cancer cells, we subsequently analysed Bcl-2 expression in NA-treated cells. As expected, downregulation of Bcl-2 expressions occurred in NA-treated $\mathrm{BCa}$ cell lines (Figure 5B). These findings prompted us to evaluate the expression levels of BAX, a pro-apoptotic protein. The ratio of Bcl-2 and BAX levels determines whether cells survive or undergo apoptosis, with a high $\mathrm{Bcl}-2 / \mathrm{BAX}$ ratio favouring survival and a low $\mathrm{Bcl}-2 / \mathrm{BAX}$ ratio favouring apoptosis. Nimocinol acetate-treatment 

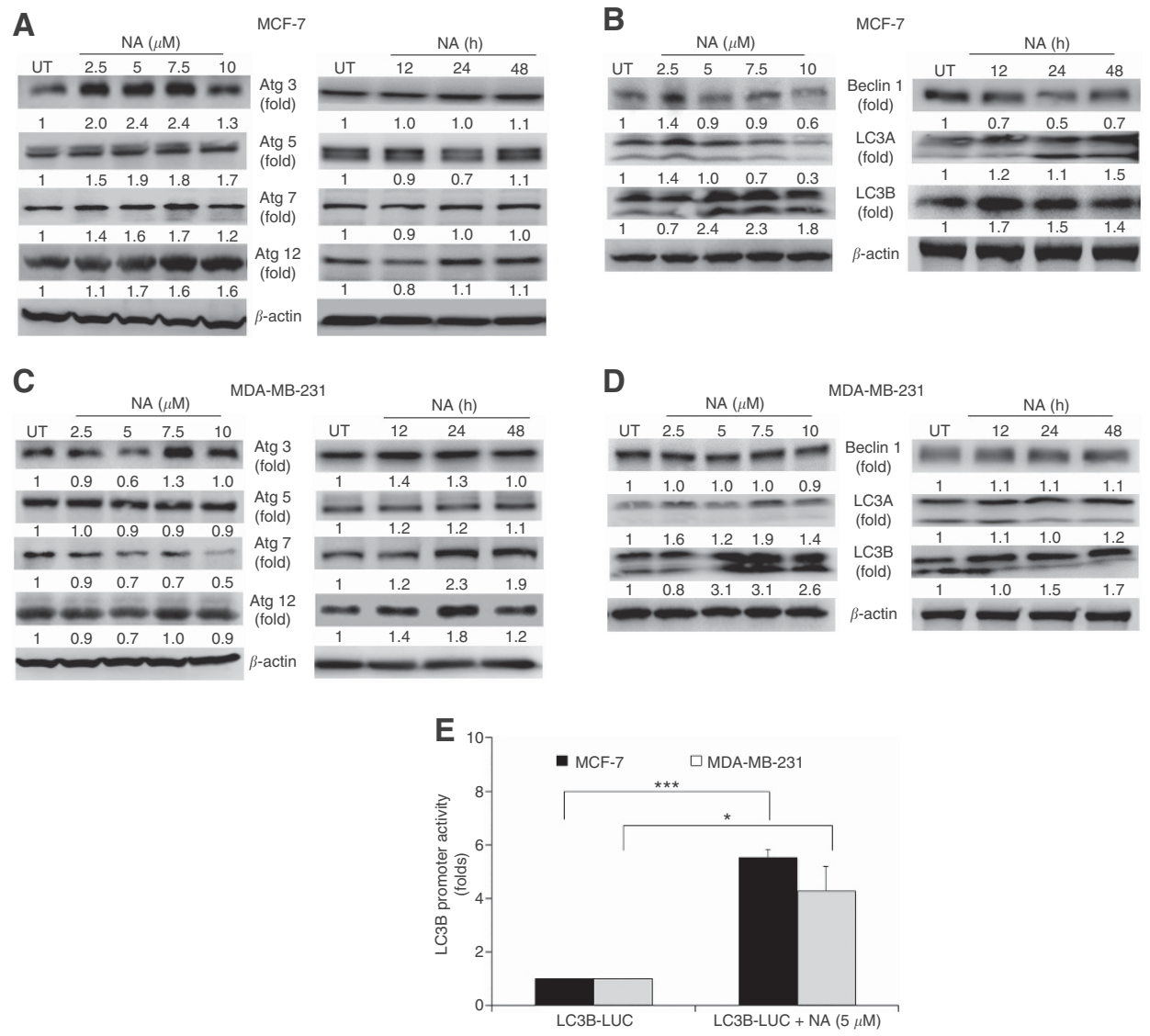

Figure 3. Nimocinol acetate induces autophagy markers in BCa cells. (A and B) To determine the dose- and time-dependent effects of NA on expression of autophagy markers in MCF-7 cells, whole-cell lysates were used for western blot analysis to determine the expression levels of Atg3, Atg5, Atg7,Atg12, Beclin 1, LC3A, and LC3B after NA treatment. (C and D) Similarly, dose- and time-dependent effects of NA on expression of autophagy markers were determined in MDA-MB-231 cells. (E) The dual-luciferase reporter assay showing LC3B promoter activity in NA-treated MCF-7 and MDA-MB-231 cells. ${ }^{*} P<0.05$ and ${ }^{\star * *} P<0.0001$

led to upregulated BAX expression in both BCa cell lines in a timedependent manner (Figure 5B). We also found that the cleaved forms of caspase-9, caspase-3, and poly (adenosine diphosphateribose) polymerase (PARP), proteins involved in apoptosis were increased in NA-treated cells. However, activation of pro-apoptotic proteins did not lead to induction of apoptosis. These observations suggest that autophagy governs the downstream pro-apoptotic effectors to execute growth arrest in BCa cells.

Induction of autophagic signalling by NA is dependent on LC3B activation. Next, we analysed whether LC3B is responsible for NA-induced autophagy in BCa cells. We transiently transfected siRNA specific for LC3B into BCa cell lines. Inhibition of LC3B significantly suppressed NA-mediated growth suppression in $\mathrm{BCa}$ cell lines (Figures 6A and B). The NA-induced autophagosome formation was attenuated in siRNA-treated $\mathrm{BCa}$ cell lines suggesting that $\mathrm{LC} 3 \mathrm{~B}$ might be a major factor required for the NA-mediated growth inhibition in BCa cells (Figures 6A and B). These data clearly suggest that autophagy is the mechanism of action of NA, and that the anti-neoplastic effects of NA in BCa are dependent on the expression and/or activation of LC3B.

\section{DISCUSSION}

Autophagy is a multistep process that is regulated by a number of signalling pathways in cancer cells. On the basis of the level of stress signalling, the role of autophagy varies from a pro-survival to a pro-apoptotic process in cancer cells. A low level of stressinduced autophagy is thought to induce cell survival and proliferation, whereas higher extents or prolonged induction of autophagy are thought to result in cancer cell death.

Our TMA results show that LC3B was highly expressed in breast tumour tissues as compared with normal breast tissues; however, no significant differences in LC3B expression were found between each stage of breast tumour. Many groups are focusing on inhibiting LC3B expression as a therapeutic option for breast cancer. However, in this study, we intent to increase the threshold of LC3B expression specifically in breast cancer cells and forcing the cells to undergo growth inhibition. Nimocinol acetate, a small molecule extracted from an edible fruit, promotes growth inhibition and exerts anticancer effects primarily by inducing autophagy in both ERpositive and ER-negative BCa cells. Like NA, dietary agents such as resveratrol and prenylated flavones, have been shown to cause growth arrest by activating autophagy signalling in BCa cells (Pedro et al, 2006; Garcia-Zepeda et al, 2013). Therefore, in cancer cells that exhibit defective pro-apoptotic signalling or resistance to apoptotic machinery, targeting autophagy-mediated cell death could be an alternative strategy for inducing cell death. In addition, developing novel molecules that activate autophagy signalling should be explored for application in cancer treatment.

Nimocinol acetate-mediated growth inhibition appears specific to BCa cells, because no significant NA-mediated toxicity was found in normal breast epithelial cells and PBMCs, which suggests that NA could be a novel chemopreventive and chemotherapeutic agent safe for use in preclinical and clinical studies. Our results also show that 

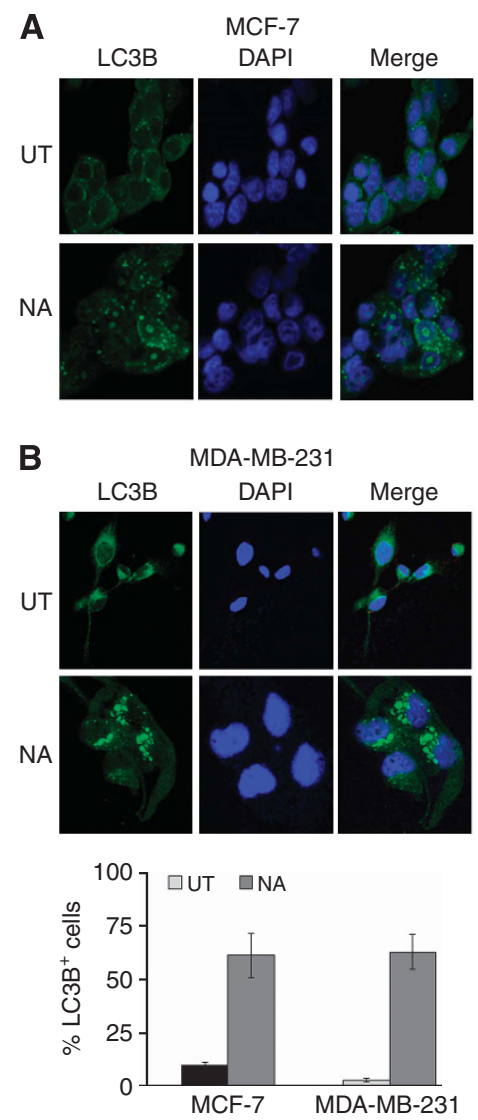

C MCF-12A MCF-7

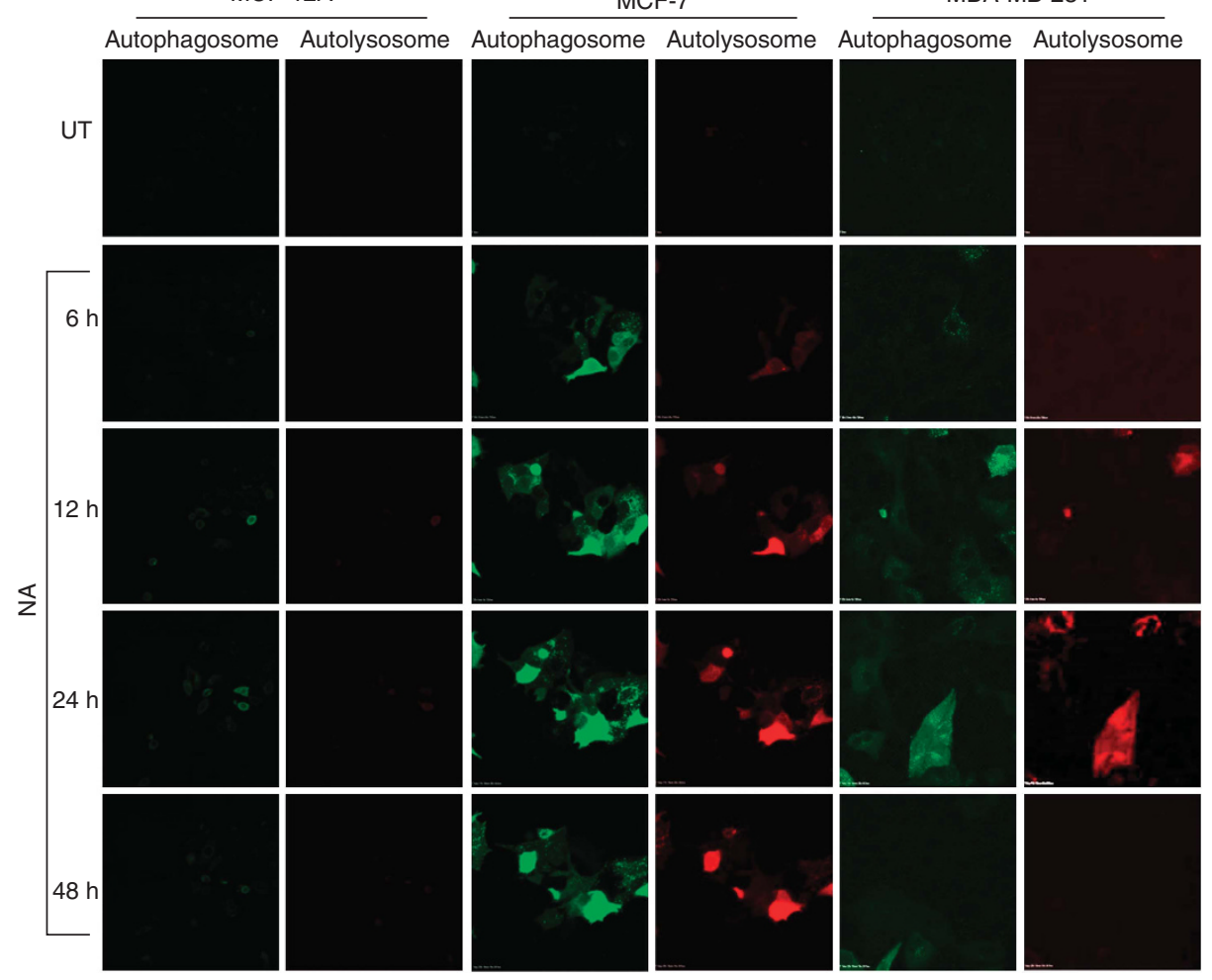

Figure 4. Nimocinol acetate induces autophagosome and autolysosome formation in BCa cells. (A and B) Confocal microscopy images showing autophagosome formation, marked by the presence of LC3B puncta. (C) MCF-12A, MCF-7, and MDA-MB-231 cells were treated with $\mathrm{NA}$ and images of the cells were captured using a live cell imaging microscope programmed to take photographs every $1 \mathrm{~h}$, for up to $48 \mathrm{~h}$. Autophagosomes, with neutral $\mathrm{pH}$, are indicated by acid-sensitive GFP emission, whereas autolysosomes, with acidic $\mathrm{pH}$, are indicated by acid-insensitive RFP emission concomitant with loss of GFP emission.

A
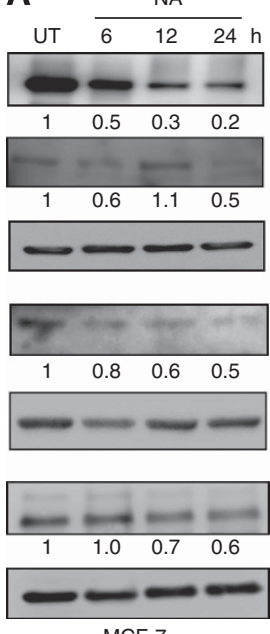

MCF-7
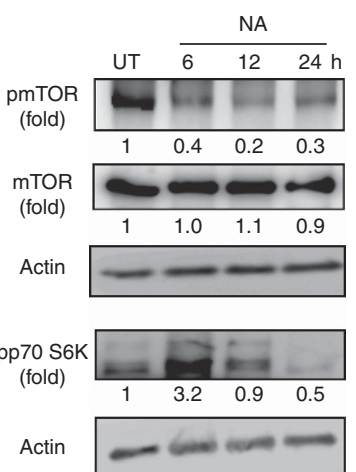

p70 S6 K (fold)

Actin

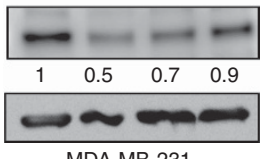

B
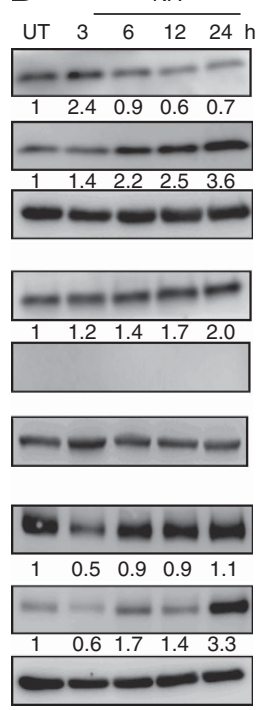

MCF-7
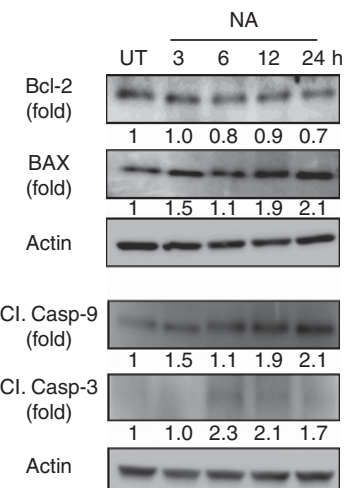

PARP

(fold)

CI. PARP (fold)

Actin

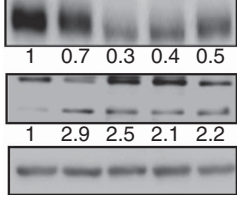

Figure 5. Nimocinol acetate inhibits mTOR signalling and induces pro-apoptotic signalling in BCa cells. (A) MCF-7 and MDA-MB-231 cells were treated with vehicle alone or NA and total cell lysates were prepared. Equal amount of proteins were used to evaluate the expression patterns of mTOR, phospho-mTOR, p70s6K, and phospho-p70s6K using western blotting analysis. (B) MCF-7 and MDA-MB-231 cells were treated with vehicle alone or NA for indicated time periods. Total cell lysates were prepared and the expression levels of Bcl-2, BAX, cleaved caspase-9 and -3, PARP, and cleaved PARP expression were detected by western blotting. 
A
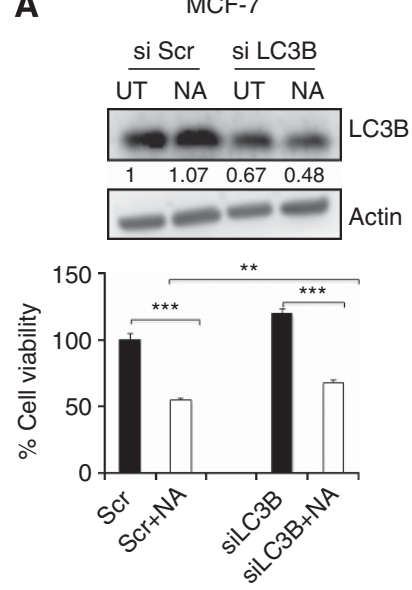

B
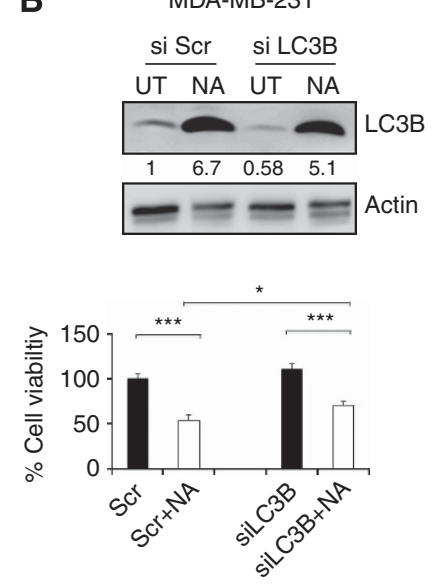

Figure 6. Nimocinol acetate-induced autophagy is LC3B-dependent. (A) MCF-7 cells were transiently transfected with scrambled control (siScr) or LC3B-specific (siLC3B) siRNA for $36 \mathrm{~h}$ followed by treatment with NA. Cells were then harvested and cell viability was measured. Whole-cell lysates were prepared and western blot was performed to assess the levels of LC3B and $\beta$-actin proteins. (B) Similarly, MDA-MB231 cells were transiently transfected with siScr or siLC3B, and cell viability and protein expression levels were measured. Data are expressed as the mean \pm s.e.m. of two independent experiments. ${ }^{\star} P<0.05,{ }^{* \star} P<0.005$ and ${ }^{\star * \star} P<0.0001$.

the use of $\mathrm{NA}$ at its $\mathrm{IC}_{50}$ concentrations did not cause either induction of apoptosis or cell cycle arrest, following treatment for up to $48 \mathrm{~h}$. The roles of autophagy and apoptosis in cancer progression are controversial; however, there is cross-talk of molecular signalling between these two events such that they share common proapoptotic signalling molecules in cancer cells (Maiuri et al, 2007).

In our results, no significant inductions of beclin 1 expression were observed in NA-treated BCa cells. Beclin 1 is thought to be an important autophagy-regulated factor and beclin 1 activation is thought to be essential for induction of autophagy in BCa cells. However, a recent study found that beclin 1 is non-functional in some BCa cells because of a mutation in one of the beclin 1 alleles (Liang et al, 1999; Qu et al, 2003). Thus, we studied other key regulators of autophagy, the ATGs, that initiate autophagosome and autolysosome formation, which eventually lead to cellular breakdown and recycling (reviewed in Eskelinen and Saftig, 2009). Nimocinol acetate induced Atg3 protein expression, as compared to $\operatorname{Atg} 7$ or Atg12 expression, in a dose- and time-dependent manner. We also saw a moderate induction of Atg5 in NA-treated MCF-7 and MDA-MB-231 cells. Activation of Atg3, Atg5, and Atg12 initiates the pre-autophagosome and promotes the conversion of LC3B-I to LC3B-II, which facilitates the formation of the double membrane of the autophagosome (reviewed in Tanida et al, 2004). The conversion of LC3B-I to LC3B-II is an indicator of autophagosome formation, and the results of the confocal microscopy analysis suggested that LC3B was localised in vacuoles (puncta) in NA-treated cells. The formation of an increased number of autophagy vacuoles in NA-treated BCa cells suggests a breakdown of cytosolic cellular components within the lysosome. In addition, kinetic analyses of autophagosome and autolysosome formation in MCF-7, MDA-MB-231, and MCF-12A cells were performed, with or without NA treatment, by live cell imaging. Initiation of autophagosome formation, followed by autolysosome formation, was prominent in BCa cells treated with NA; however, induction of autophagosome/autolysosome formation was less pronounced in normal breast epithelial cells treated with NA. Formation of autophagosomes is an early event in autophagy. The fusion of autophagosomes with lysosomes (i.e., formation of autolysosomes) is a critical step in initiation of cell death (Trincheri et al, 2008). The degradation of the lysosome has been suggested to be the determining event in induction of autophagy- and necrosismediated cell death (Kroemer and Jaattela, 2005).

Activation of mTOR signalling negatively regulates autophagy by upregulating phosphorylation of Atg13, that in turn inhibits the formation of a trimeric complex with ULK1, which, in turn, is required for formation of the autophagosome (Yang et al, 2011). Therefore, we analysed whether NA inhibits the mTOR pathway. Our results confirmed that NA downregulates phospho-mTOR and p70S6K, suggesting that inhibition of the mTOR pathway may be a mechanism of NA-mediated autophagy in BCa cells. Multiple studies also suggested that rapamycin, a specific inhibitor of mTOR, induces autophagy in several cancer cell lines (Hashimoto et al, 2008; Kim et al, 2008, 2009). There are many signalling molecules that negatively regulate autophagy, and $\mathrm{Bcl}-2$ and $\mathrm{BCL}_{\mathrm{XL}}$ are pro-survival proteins (Oh et al, 2011) that form a complex with beclin 1 , thereby inhibiting its functions in autophagy (Fimia and Piacentini, 2010). Phosphorylation of Bcl-2 and $\mathrm{BCL}_{\mathrm{xL}}$ by $\mathrm{c}-\mathrm{Jun}$ $\mathrm{N}$-terminal kinase disrupts the association with beclin 1 , and the release of beclin1 activates autophagy-mediated cell death (Wei et al, 2008; Zhou et al, 2011). Our results show that NA inhibits the expression of Bcl-2 in both MCF-7 and MDA-MB-231 cells, suggesting that NA activates both autophagic and apoptotic signals in these cells. Moreover, the upregulation of BAX expression in NA-treated BCa cells suggests initiation of caspase-9-mediated apoptotic signalling. It has been reported that most of the BCa cell lines exhibit low levels of caspase activation, although caspase- 3 is deficient in MCF-7 cells (Yang et al, 2007). Devarajan et al (2002) reported downregulation of caspse- 3 mRNA expression in $75 \%$ of $\mathrm{BCa}$ samples and suggested that this downregulation may be responsible for cancer cell survival and chemoresistance, consistent with data reported in another study (Nassar et al, 2008). The results herein show induction of caspase- 9 and caspase-3, along with PARP cleavage, in NA-treated BCa cells, suggesting that NA activates autophagy-mediated cell death signalling in BCa cells.

In conclusion, LC3B is highly expressed in breast tumour tissues and increasing threshold of LC3B activation forces the BCa cells undergo growth inhibition. Nimocinol acetate appears to be a potent herbal molecule that inhibits the growth of both ER-positive and ER-negative BCa cells, without causing toxicity to normal breast epithelial cells. The role of autophagy in cancer, especially the link between autophagy and apoptosis, is quite complex; however, our results suggest that treatment with NA induces autophagy and promotes growth inhibition in both ER-positive and -negative $\mathrm{BCa}$ cells. Determining the in vivo efficacy of NA, either as a therapy or for prevention, in BCa models may help uncover additional targets of NA. Our data support the need for further studies to address the mechanisms underlying the effects of $\mathrm{NA}$ on $\mathrm{BCa}$ and to evaluate the utility and application of NA in the clinical setting.

\section{CONFLICT OF INTEREST}

The authors declare no conflict of interest.

\section{REFERENCES}

Abedin MJ, Wang D, McDonnell MA, Lehmann U, Kelekar A (2007) Autophagy delays apoptotic death in breast cancer cells following DNA damage. Cell Death Differ 14(3): 500-510.

Chen RJ, Tsai SJ, Ho CT, Pan MH, Ho YS, Wu CH, Wang YJ (2012) Chemopreventive effects of pterostilbene on urethane-induced lung carcinogenesis in mice via the inhibition of EGFR-mediated pathways and the induction of apoptosis and autophagy. J Agric Food Chem 60(46): 11533-11541. 
Das TP, Suman S, Damodaran C (2013) Reactive oxygen species generation inhibits epithelial-mesenchymal transition and promotes growth arrest in prostate cancer cells. Mol Carcinog; e-pub ahead of print 8 March 2013; doi:10.1002/mc.22014.

Devarajan E, Sahin AA, Chen JS, Krishnamurthy RR, Aggarwal N, Brun AM, Sapino A, Zhang F, Sharma D, Yang XH, Tora AD, Mehta K (2002) Down-regulation of caspase 3 in breast cancer: a possible mechanism for chemoresistance. Oncogene 21(57): 8843-8851.

Eskelinen EL, Saftig P (2009) Autophagy: a lysosomal degradation pathway with a central role in health and disease. Biochim Biophys Acta 1793(4): 664-673.

Fimia GM, Piacentini M (2010) Regulation of autophagy in mammals and its interplay with apoptosis. Cell Mol Life Sci 67(10): 1581-1588.

Furuya N, Yu J, Byfield M, Pattingre S, Levine B (2005) The evolutionarily conserved domain of Beclin 1 is required for Vps34 binding, autophagy and tumor suppressor function. Autophagy 1(1): 46-52.

Futreal PA, Soderkvist P, Marks JR, Iglehart JD, Cochran C, Barrett JC, Wiseman RW (1992) Detection of frequent allelic loss on proximal chromosome $17 \mathrm{q}$ in sporadic breast carcinoma using microsatellite length polymorphisms. Cancer Res 52(9): 2624-2627.

Garcia-Zepeda SP, Garcia-Villa E, Diaz-Chavez J, Hernandez-Pando R, Gariglio P (2013) Resveratrol induces cell death in cervical cancer cells through apoptosis and autophagy. Eur J Cancer Prev 22(6): 577-584.

Hara T, Takamura A, Kishi C, Iemura S, Natsume T, Guan JL, Mizushima N (2008) FIP200, a ULK-interacting protein, is required for autophagosome formation in mammalian cells. J Cell Biol 181(3): 497-510.

Hashimoto I, Koizumi K, Tatematsu M, Minami T, Cho S, Takeno N, Nakashima A, Sakurai H, Saito S, Tsukada K, Saiki I (2008) Blocking on the CXCR4/mTOR signalling pathway induces the anti-metastatic properties and autophagic cell death in peritoneal disseminated gastric cancer cells. Eur J Cancer 44(7): 1022-1029.

Hosokawa N, Sasaki T, Iemura S, Natsume T, Hara T, Mizushima N (2009) Atg101, a novel mammalian autophagy protein interacting with Atg13. Autophagy 5(7): 973-979.

Jung CH, Jun CB, Ro SH, Kim YM, Otto NM, Cao J, Kundu M, Kim DH (2009) ULK-Atg13-FIP200 complexes mediate mTOR signaling to the autophagy machinery. Mol Biol Cell 20(7): 1992-2003.

Kabeya Y, Mizushima N, Ueno T, Yamamoto A, Kirisako T, Noda T, Kominami E, Ohsumi Y, Yoshimori T (2000) LC3, a mammalian homologue of yeast Apg8p, is localized in autophagosome membranes after processing. EMBO J 19(21): 5720-5728.

Kanzawa T, Germano IM, Komata T, Ito H, Kondo Y, Kondo S (2004) Role of autophagy in temozolomide-induced cytotoxicity for malignant glioma cells. Cell Death Differ 11(4): 448-457.

Kim DK, Yang JS, Maiti K, Hwang JI, Kim K, Seen D, Ahn Y, Lee C, Kang BC, Kwon HB, Cheon J, Seong JY (2009) A gonadotropin-releasing hormone-II antagonist induces autophagy of prostate cancer cells. Cancer Res 69(3): 923-931.

Kim KW, Hwang M, Moretti L, Jaboin JJ, Cha YI, Lu B (2008) Autophagy upregulation by inhibitors of caspase- 3 and $\mathrm{mTOR}$ enhances radiotherapy in a mouse model of lung cancer. Autophagy 4(5): 659-668.

Klionsky DJ, Emr SD (2000) Autophagy as a regulated pathway of cellular degradation. Science 290(5497): 1717-1721.

Kondo Y, Kondo S (2006) Autophagy and cancer therapy. Autophagy 2(2): 85-90.

Kroemer G, Jaattela M (2005) Lysosomes and autophagy in cell death control. Nat Rev Cancer 5(11): 886-897.

Kroemer G, Levine B (2008) Autophagic cell death: the story of a misnomer. Nat Rev Mol Cell Biol 9(12): 1004-1010.

Laane E, Tamm KP, Buentke E, Ito K, Kharaziha P, Oscarsson J, Corcoran M, Bjorklund AC, Hultenby K, Lundin J, Heyman M, Soderhall S, Mazur J, Porwit A, Pandolfi PP, Zhivotovsky B, Panaretakis T, Grander D (2009) Cell death induced by dexamethasone in lymphoid leukemia is mediated through initiation of autophagy. Cell Death Differ 16(7): 1018-1029.

Li J, Hou N, Faried A, Tsutsumi S, Kuwano H (2010) Inhibition of autophagy augments 5-fluorouracil chemotherapy in human colon cancer in vitro and in vivo model. Eur J Cancer 46(10): 1900-1909.

Liang XH, Jackson S, Seaman M, Brown K, Kempkes B, Hibshoosh H, Levine B (1999) Induction of autophagy and inhibition of tumorigenesis by beclin 1 . Nature 402(6762): 672-676.

Lum JJ, Bauer DE, Kong M, Harris MH, Li C, Lindsten T, Thompson CB (2005) Growth factor regulation of autophagy and cell survival in the absence of apoptosis. Cell 120(2): 237-248.

Maiuri MC, Zalckvar E, Kimchi A, Kroemer G (2007) Self-eating and selfkilling: crosstalk between autophagy and apoptosis. Nat Rev Mol Cell Biol 8(9): 741-752.
Miller BC, Zhao Z, Stephenson LM, Cadwell K, Pua HH, Lee HK, Mizushima NN, Iwasaki A, He YW, Swat W, Virgin HWt (2008) The autophagy gene ATG5 plays an essential role in B lymphocyte development. Autophagy 4(3): 309-314

Miracco C, Cosci E, Oliveri G, Luzi P, Pacenti L, Monciatti I, Mannucci S, De Nisi MC, Toscano M, Malagnino V, Falzarano SM, Pirtoli L, Tosi P (2007) Protein and mRNA expression of autophagy gene Beclin 1 in human brain tumours. Int J Oncol 30(2): 429-436.

Mizushima N, Yoshimori T (2007) How to interpret LC3 immunoblotting. Autophagy 3(6): 542-545.

Nassar A, Lawson D, Cotsonis G, Cohen C (2008) Survivin and caspase-3 expression in breast cancer: correlation with prognostic parameters, proliferation, angiogenesis, and outcome. Appl Immunohistochem Mol Morphol 16(2): 113-120.

Oh S, Xiaofei E, Ni D, Pirooz SD, Lee JY, Lee D, Zhao Z, Lee S, Lee H, Ku B, Kowalik T, Martin SE, Oh BH, Jung JU, Liang C (2011) Downregulation of autophagy by Bcl-2 promotes MCF7 breast cancer cell growth independent of its inhibition of apoptosis. Cell Death Differ 18(3): 452-464.

Paglin S, Hollister T, Delohery T, Hackett N, McMahill M, Sphicas E, Domingo D, Yahalom J (2001) A novel response of cancer cells to radiation involves autophagy and formation of acidic vesicles. Cancer Res 61(2): 439-444.

Pattingre S, Tassa A, Qu X, Garuti R, Liang XH, Mizushima N, Packer M, Schneider MD, Levine B (2005) Bcl-2 antiapoptotic proteins inhibit Beclin 1-dependent autophagy. Cell 122(6): 927-939.

Pedro M, Lourenco CF, Cidade H, Kijjoa A, Pinto M, Nascimento MS (2006) Effects of natural prenylated flavones in the phenotypical ER (+) MCF-7 and ER ( - ) MDA-MB-231 human breast cancer cells. Toxicol Lett 164(1): 24-36.

Qu X, Yu J, Bhagat G, Furuya N, Hibshoosh H, Troxel A, Rosen J, Eskelinen EL, Mizushima N, Ohsumi Y, Cattoretti G, Levine B (2003) Promotion of tumorigenesis by heterozygous disruption of the beclin 1 autophagy gene. J Clin Invest 112(12): 1809-1820.

Ravikumar B, Vacher C, Berger Z, Davies JE, Luo S, Oroz LG, Scaravilli F, Easton DF, Duden R, O'Kane CJ, Rubinsztein DC (2004) Inhibition of mTOR induces autophagy and reduces toxicity of polyglutamine expansions in fly and mouse models of Huntington disease. Nat Genet 36(6): 585-595.

Ren JH, He WS, Nong L, Zhu QY, Hu K, Zhang RG, Huang LL, Zhu F, Wu G (2010) Acquired cisplatin resistance in human lung adenocarcinoma cells is associated with enhanced autophagy. Cancer Biother Radiopharm 25(1): $75-80$.

Russell SE, Hickey GI, Lowry WS, White P, Atkinson RJ (1990) Allele loss from chromosome 17 in ovarian cancer. Oncogene 5(10): 1581-1583.

Sharma N, Thomas S, Golden EB, Hofman FM, Chen TC, Petasis NA, Schonthal AH, Louie SG (2012) Inhibition of autophagy and induction of breast cancer cell death by mefloquine, an antimalarial agent. Cancer Lett 326(2): 143-154.

Shi WY, Xiao D, Wang L, Dong LH, Yan ZX, Shen ZX, Chen SJ, Chen Y, Zhao WL (2012) Therapeutic metformin/AMPK activation blocked lymphoma cell growth via inhibition of mTOR pathway and induction of autophagy. Cell Death Dis 3: e275.

Siegel R, Naishadham D, Jemal A (2013) Cancer statistics, 2013. CA Cancer J Clin 63(1): 11-30.

Suman S, Das TP, Damodaran C (2013a) Silencing NOTCH signaling causes growth arrest in both breast cancer stem cells and breast cancer cells. Br J Cancer 109(10): 2587-2596.

Suman S, Kurisetty V, Das TP, Vadodkar A, Ramos G, Lakshmanaswamy R, Damodaran C (2013b) Activation of AKT signaling promotes epithelialmesenchymal transition and tumor growth in colorectal cancer cells. Mol Carcinog 53(Suppl 1): E151-E160.

Tanida I, Ueno T, Kominami E (2004) LC3 conjugation system in mammalian autophagy. Int J Biochem Cell Biol 36(12): 2503-2518.

Trincheri NF, Follo C, Nicotra G, Peracchio C, Castino R, Isidoro C (2008) Resveratrol-induced apoptosis depends on the lipid kinase activity of Vps34 and on the formation of autophagolysosomes. Carcinogenesis 29(2): 381-389.

Wei Y, Pattingre S, Sinha S, Bassik M, Levine B (2008) JNK1-mediated phosphorylation of Bcl-2 regulates starvation-induced autophagy. Mol Cell 30(6): 678-688.

Yang PM, Tseng HH, Peng CW, Chen WS, Chiu SJ (2012) Dietary flavonoid fisetin targets caspase-3-deficient human breast cancer MCF-7 cells by 
induction of caspase-7-associated apoptosis and inhibition of autophagy. Int J Oncol 40(2): 469-478.

Yang S, Liu J, Thor AD, Yang X (2007) Caspase expression profile and functional activity in a panel of breast cancer cell lines. Oncol Rep 17(5): 1229-1235.

Yang ZJ, Chee CE, Huang S, Sinicrope FA (2011) The role of autophagy in cancer: therapeutic implications. Mol Cancer Ther 10(9): 1533-1541.

Zeng X, Kinsella TJ (2008) Mammalian target of rapamycin and S6 kinase 1 positively regulate 6-thioguanine-induced autophagy. Cancer Res 68(7): 2384-2390.
Zhou F, Yang Y, Xing D (2011) Bcl-2 and Bcl-xL play important roles in the crosstalk between autophagy and apoptosis. FEBS J 278(3): 403-413.

This work is published under the standard license to publish agreement. After 12 months the work will become freely available and the license terms will switch to a Creative Commons AttributionNonCommercial-Share Alike 3.0 Unported License.

Supplementary Information accompanies this paper on British Journal of Cancer website (http://www.nature.com/bjc) 